\title{
EchoGéo
}

$53 \mid 2020$

Dénominations plurielles. Quand les noms de lieux se concurrencent

\section{Révéler les traces du toxique}

Benjamin Lysaniuk, Anaïs Ondet et Léa Prost

\section{(2) OpenEdition}

Journals

Édition électronique

URL : https://journals.openedition.org/echogeo/19792

DOI : 10.4000/echogeo. 19792

ISSN : 1963-1197

Éditeur

Pôle de recherche pour l'organisation et la diffusion de l'information géographique (CNRS UMR 8586)

Référence électronique

Benjamin Lysaniuk, Anaïs Ondet et Léa Prost, «Révéler les traces du toxique », EchoGéo [En ligne], 53 | 2020, mis en ligne le 20 octobre 2020, consulté le 11 août 2021. URL : http://journals.openedition.org/ echogeo/19792 ; DOI : https://doi.org/10.4000/echogeo.19792

Ce document a été généré automatiquement le 11 août 2021.

EchoGéo est mis à disposition selon les termes de la licence Creative Commons Attribution - Pas d'Utilisation Commerciale - Pas de Modification 4.0 International (CC BY-NC-ND) 


\title{
Révéler les traces du toxique
}

\author{
Benjamin Lysaniuk, Anaïs Ondet et Léa Prost
}

Cet article poursuit les réflexions menées dans le cadre d'un séminaire organisé par l'axe 1 de l'EA 7338 Pléiade (Université Paris 13, Sorbonne Paris Cité) en juin 2019 « Images des marges. Quels apports des sciences humaines et sociales?». Cette séance consacrée aux usages de la photographie par des chercheur.e.s en sciences sociales nous a offert la possibilité d'instaurer un dialogue " géographie-photographie » autour de la thématique des impacts sanitaires et environnementaux des agropesticides à partir de la série photographique Les mauvaises herbes. Les auteurs de l'article sont cités par ordre alphabétique, ayant contribué à parts égales à sa rédaction.

1 Les mauvaises herbes est un documentaire qui questionne la responsabilité des pesticides dans la survenue de maladies chez des femmes et des hommes en contact avec les agropesticides. Ce travail fut réalisé de 2018 à 2019 sur le territoire français, en collaboration avec l'association Phyto-Victimes, qui a rendue possible la rencontre de la photographe avec les victimes. Il se compose d'une série de portraits avec entretiens enregistrés et d'une série de paysages.

2 En endossant le rôle de porte-voix, l'artiste ambitionne de mettre en lumière les conséquences sanitaires d'un modèle agricole mortifère aussi productif soit-il. Son travail permet de lire aussi bien la photographie comme trace que les traces sur la photographie : il agit comme un révélateur des toxiques et de leurs impacts. À partir de deux photographies issues de ce documentaire et des commentaires associés, nous nous interrogerons sur la manière dont un procédé artistique contribue à rompre l'invisibilité des expositions pathogènes et de leurs victimes. Le travail de contextualisation lié à ces photographies semble, en ce sens, revêtir une importance fondamentale. La portée politique du message véhiculé par l'artiste est évidente : elle situe ce travail en contrepoids des mécanismes connus de production d'ignorance (science orientée ou non produite) qui gomment les pollutions et leurs conséquences.

3 Cette contribution démontre une nouvelle fois l'intérêt du discours photographique et de son décryptage par les sciences humaines et sociales et leur capacité à analyser les rapports de pouvoir dans le champ des controverses scientifiques. Elle illustre enfin 
l'aptitude des géographes à saisir les dynamiques paysagères y compris à partir d'une portion de territoire figée sur une image.

\section{Christian}

«On m'a dit: 'vous avez un parkinson atypique' [...] parce que je tremble pas, j’ai pas tous les symptômes. Mais on m'a dit, c'est une maladie 'sans retour' ». Christian, 72 ans, viticulteur.

\section{Illustration 1 - Christian, 72 ans, atteint de la maladie de Parkinson}

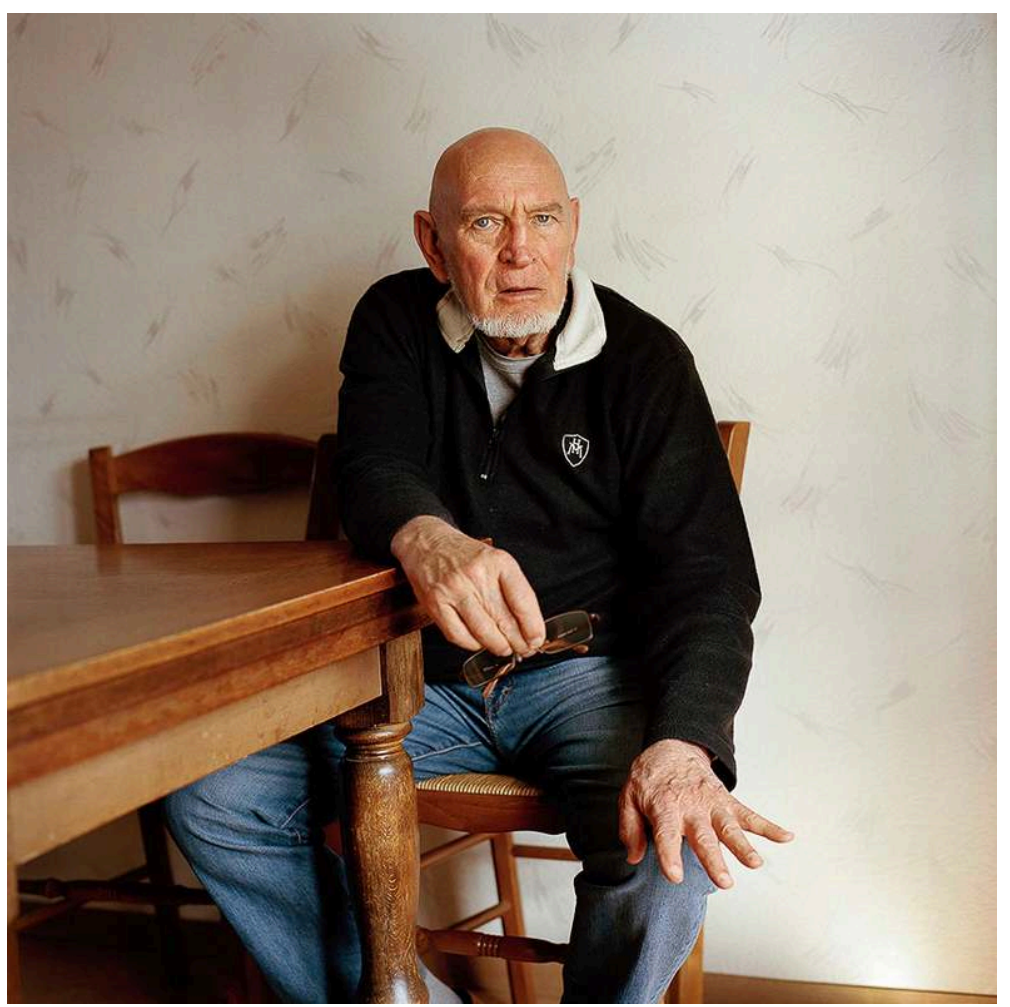

Auteure : Anaiis Ondet.

Nous avons choisi, ici, le portrait d'un homme : Christian. C'est une photographie au format carré avec un angle frontal et à hauteur des yeux de l'homme. Il est assis sur une chaise en bois, accoudé à une table, elle aussi en bois. On peut deviner qu'il est chez lui dans sa salle à manger, dans son intimité. Le décor est très simple et épuré : une table, deux chaises et un mur avec un papier peint blanc aux motifs fins et éparses. Le portrait apparaît somme-toute banal, l'homme aux yeux très bleus, a un visage assez impassible, néanmoins nous nous sentons transpercés par son regard. Puis nos yeux finissent par s'abaisser et s'attarder sur ses mains, suspendues. L'une tient une paire de lunettes, l'autre, légèrement appuyée sur la cuisse, est complètement ouverte et en tension. On sent alors une étrange rigidité dans le corps de cet homme, qui nous interroge. Son regard et la position de sa main produisent un effet d'inquiétante étrangeté ${ }^{1}$. Cette personne est atteinte de la maladie de Parkinson du fait de l'utilisation de produits phytosanitaires au cours de son activité viticole.

Toute étude d'un sujet nécessite un support. Arts visuels, littérature, poésie, etc... En 2018, je décide de travailler sur la problématique des pesticides par la photographie, en débutant Les mauvaises herbes. Le photojournalisme qui se caractérise par un nombre conséquent d'images d'actualités, prises lors d'événements particuliers, à des fins de communication, m'apparaît obsolète car, 
dans la société actuelle où nous sommes baignés dans les images, ces photographies se noient entre elles par leur nombre et sont malheureusement souvent dépendantes des lignes éditoriales. Comment, alors, contourner le photojournalisme pour traiter de sujets sociaux et environnementaux? L'approche documentaire m'est apparue la plus propice pour ce sujet sur les pesticides. Cette démarche se veut en rupture avec l'héritage classique du photojournalisme. Soulages (2017, p. 208) suggère que « cette autre manière de faire et d'être se repère dans un autre rapport au temps : ces photographes ne cherchent plus à capturer l'instant; ils s'installent et s'interrogent délibérément dans le temps: dans la longue durée avec la photographie comme enquête, dans le passé avec la photographie comme mémoire, dans le temps intersubjectif avec la photographie comme interaction». Poussée par ma double sensibilité photographique et politique, j'ai alors pris le temps de créer un documentaire; je suis allée creuser dans cette crise sanitaire, sociale et écologique. Le sujet des pesticides était un prétexte à créer et à produire une série, mais la photographie est également devenue un prétexte pour enquêter et apprendre sur ce sujet. En effet, bien que concernée par les problématiques environnementales actuelles, force est de constater que j'avais une sensation d'impuissance et ne trouvais pas de formes concrètes et efficaces pour matérialiser mon engagement militant. J'espère, par la diffusion de mes images, pouvoir toucher un public large, qui n'est peut-être pas encore sensibilisé à ces thématiques. La photographie est ainsi, dans ma démarche, autant un acte artistique que politique.

Les mauvaises herbes est un corpus d'images et de témoignages contemporains de femmes et d'hommes que l'État et les multinationales tentent de résoudre au silence. En découle ainsi un projet doublement artistique et politique qui tend à dénoncer les dérives et la dangerosité de l'industrie agrochimiques et sert de témoignage de ce scandale sanitaire tout en en organisant la conservation de traces. Ma démarche photographique peut être rapprochée de la première fonction de la photographie, qui était tout d'abord orientée vers le portrait et qui servait d'élévation sociale et de représentation pour la petite bourgeoisie et de la classe moyenne qui n'avaient alors jusque-là peu de moyen de représentation. Freund (1974, p. 11) écrivait en ce sens que " 'faire faire son portrait' était un de ces actes symboliques par lesquels les individus de la classe sociale ascendante rendaient visible à eux-mêmes et aux autres leur ascension et se classaient parmi ceux qui jouissaient de la considération sociale ». Par ce travail de portrait de victimes de pesticides, je choisis de donner une représentation à des sans-voix, des sans-visage et ainsi de rendre visible une problématique qui n'est peu, voire pas reconnue.

Dans ce travail documentaire sur les conséquences sanitaires des pesticides, avant de réaliser le portrait des personnes rencontrées, je réalise un entretien semidirectif afin d'en savoir plus sur leur parcours professionnels et personnels ainsi que sur leur maladie. Cet entretien, avant la prise photographique, me permet d'en apprendre plus sur les pesticides et leurs effets, mais aussi, de créer un lien avec cette personne. Je peux ainsi, en tant que photographe, mieux cerner la personnalité et la sensibilité de mon sujet la personne en face de moi avant de le la photographier. Puis l'installation, longue, de l'appareil photo, un moyen format Hasselblad sur trépied, me permet de discuter encore et surtout de préparer la personne à la photographie. J'attache une grande importance dans ma démarche à prendre le temps pour la prise de vue. Faire le portrait de quelqu'un c'est d'une certaine façon prendre une chose très intime de cette personne. C'est fixer son image et figer cet instant. Capter quelque chose qu'elle n'aura jamais l'occasion de voir réellement. Ce type de prise de vue est pour moi un processus assez lent et calme, qui s'inscrit dans une volonté de mettre les personnes dans une situation confortable afin qu'au bout de quelques minutes, une expression sur leur visage, un geste, émerge et m'offre ee un "creux» dans la posture dans laquelle se met le photographié qui me livre finalement un fragment d'intimité. J'espère aussi, sûrement, que mon attitude empathique se traduira in fine dans les photographies 
présentées afin qu'elles puissent à leur tour rencontrer un regard empathique et venir pointer les spectateurs.

Sontag (1977, p. 35) écrit que «la télévision charrie un [tel] flux d'images dont chacune annule la précédente ». Nous sommes tellement nourris d'images que nous ne les regardons plus, les photographies nous glissent souvent dessus sans que nous n'y prêtions la moindre attention. La photographie peut mettre en lumière un phénomène invisibilisé. Néanmoins comme le souligne Susan Sontag la photographie à elle seule ne peut changer l'histoire, ni engager un mouvement. "Mais si les Américains ont eu accès à des photos montrant les souffrances des Vietnamiens [...] c'est parce que les journalistes se sentaient appuyés dans les efforts qu'ils faisaient pour se procurer ces photos, du fait que l'événement avait été défini par une fraction significative de la population comme guerre coloniale sauvage. La guerre de Corée avait été comprise différemment, comme un épisode de la juste lutte du Monde libre contre l'Union soviétique et la Chine » (Sontag, 1977, p. 35-36). Les images ont nécessairement besoin d'un contexte politique et social qui puisse les accueillir. Ainsi Les mauvaises herbes ne peut trouver un écho actuellement que grâce à l'intérêt global porté à la problématique environnementale qui va crescendo. Cet intérêt a été attisé par les nombreuses recherches documentaires réalisées et la médiatisation de ces problématiques et montre ainsi les relations possibles entre les sciences sociales et la photographie.

La photographie en tant que trace, en tant que ça a été barthien, peut être un support de mémoire, individuelle et collective. Elle comporte une double fonction de révéler ce qui n'est pas connu et d'en garder des traces. Comme le prouvent des scandales sanitaires passés (l'agent orange par exemple), en l'absence de traces (écrites ou visuelles) un phénomène peut tomber dans l'oubli. Néanmoins le photographe peut travailler sous la forme d'une enquête rétrospective qui va chercher des images d'archives individuelles afin de produire une mémoire collective. À ce titre le travail de Mathieu Asselin (2017) Monsanto: une Enquête Photographique est remarquable. Pour retracer une histoire des conséquences sanitaires du géant de l'agrochimie Monsanto, Mathieu Asselin recueille un ensemble de traces : traces physiques sur les descendants des personnes exposées aux produits pathogènes, photographies d'archives, objets d'études scientifiques etc., afin de produire un ouvrage dénonciateur mais également un objet de mémoire collective des victimes de produits agrochimiques. Ainsi dans la continuité de ces travaux d'enquête, je tente à mon tour de construire des traces des victimes des pesticides. Tout en donnant un visage à ces personnes invisibilisées, je dénonce les activités agrochimiques des multinationales. J'espère ainsi que ces femmes et ces hommes, victimes de ce modèle agricole, ne tombent pas dans l'oubli.

\section{Reconnaitre les « sans-visage »}

4 Les patients atteints de maladies professionnelles liées à une exposition aux agropesticides sont invisibles. Cette situation met en évidence le lien qui se noue dans le triptyque visibilité - reconnaissance - connaissance. En effet, il ne peut y avoir de connaissance(s) sans reconnaissance institutionnelle et il ne peut y avoir de mise en visibilité d'un problème sans connaissance(s) préalable(s). Le déficit initial de reconnaissance des victimes par les institutions semble donc être une des clés permettant d'expliquer l'invisibilité des conséquences sanitaires liées à l'exposition à des agents pathogènes d'origine industrielle utilisés massivement dans l'agriculture. En France, aujourd'hui, seules deux pathologies peuvent permettre à un agriculteur de prétendre à une reconnaissance en maladie professionnelle liée à une exposition à des agropesticides: le Lymphome Non-Hodgkinien ( $\mathrm{LNH}$ ) et la maladie de Parkinson. Lorsque l'on sait «le parcours du combattant ${ }^{2}$ " de la reconnaissance en maladie professionnelle ; lorsque l'on connait les modalités d'inscription d'une maladie dans un 
tableau de reconnaissance conséquence d'une véritable négociation ${ }^{3}$; lorsque l'on se rend compte de l'importance de dispositifs comme le Groupement d'Intérêt Scientifique sur les Cancers d'Origine Professionnelle dans le Vaucluse (Hunsmann et Lysaniuk, 2019) dans l'accompagnement dans des démarches de reconnaissance en maladie professionnelle de patients atteints - entre autres pathologies - de LNH, enfin, lorsque l'INSERM (2013) évoque deux autres maladies neurodégénératives et sept autres néoplasmes en lien avec de telles expositions : les 25 cancers et 36 maladies de Parkinson reconnus en 2016 par la Mutualité Sociale Agricole démontrent implacablement l'invisibilisation mécanique des patients-victimes par le déficit de reconnaissance institutionnelle de ces maladies résultant d'expositions pathogènes. Dès lors, si la visibilité de ces victimes ne peut - pour l'heure - pas ou rarement compter sur la reconnaissance c'est parce que celle-ci est « l'expression d'une perception évaluative dans laquelle la valeur de l'individu est 'directement' donnée » (Honneth, 2005, p. 55) : « faible » valeur théorique et froide qui renvoie nécessairement à un rapport dominantdominé, s'exprimant avec acuité dans la division sociale du travail et des risques. C'est l'invisibilisation sociale, à laquelle s'ajoutent l'ignorance toxique et l'invisibilité chimique, mise en lumière par Thébaud-Mony (2008) pour expliquer la faible reconnaissance des cancers d'origine professionnelle qui s'exprime ici. En conséquence, il convient de mobiliser d'autres ressorts pour rendre visibles ces victimes : à ce titre, Les mauvaises herbes d'Anaïs Ondet demeure exemplaire.

5 Tardy (2007) nous rappelle que la visibilité est la simple qualité pour un objet d'être vu, plus ou moins nettement, dans un espace donné. Il postule, par ailleurs, un lien fondamental entre visibilité et pouvoir, conséquence de choix politiques faisant écho au processus de reconnaissance en maladie professionnelle préalablement évoqué. « N'est pas visible ce qui n'est pas digne d'être remarqué » (Tardy, 2007, p. 20-21). Cette valeur, cette possibilité d'être remarqué sont invariablement des constructions politiques. Ainsi, des expériences artistiques visant à palier un manque identifié dans la reconnaissance institutionnelle de ces maladies permettent aussi cette maïeutique en inversant d'une certaine manière le processus cognitif : c'est la mise en visibilité de la victime qui ouvre alors la porte de la connaissance de la maladie. À ce titre, la photographie doit être considérée comme une «transformation majeure de la société car cet art permettait aux plus humbles d'accéder à la visibilité » (Tardy, 2007, p. 21). C'est précisément l'objectif poursuivi par Anaïs Ondet tel qu'elle le décrit plus haut. Sa démarche procède d'une visibilité médiatisée que Voirol (2005) conçoit comme une relation entre une portion du monde perçue par un médiateur et objectivée dans des supports (ici la photographie). Ici, « [l'artiste] traduit sa manière de voir une situation singulière et l'objective sous forme de récit » (Voirol, 2005, p. 98). La photographe insère sa démarche dans un processus de revendication de visibilité somme toute assez classique dans le domaine des mouvements sociaux: elle propose un portrait pour évoquer en miroir les sans-visage. En ce sens, l'invisibilité des victimes de pesticides ne renvoie pas seulement à « un fait cognitif, mais doit bien plutôt signifier une situation sociale particulière » (Honneth, 2005, p. 43) : invisibiliser, c'est finalement nier la valeur sociale. Dans la reconnaissance ainsi proposée par le portrait, l'artiste cherche d'une certaine manière à provoquer des réactions pour « rendre justice " (Honneth, 2005, p. 48) au sujet, là où la reconnaissance institutionnelle fait malheureusement très souvent défaut. 
6 La démarche d'Anaïs Ondet s'inscrit donc - et de manière revendiquée - dans une forme d'engagement. Elle convoque une volonté de prise de conscience et de mise en visibilité à contre-courant de forces opposées - comme celles des grandes entreprises productrices de produits phytopharmaceutiques et de leur intense travail de lobbying auprès des parlementaires des grands pays agricoles - vis-à-vis desquelles l'Histoire démontre qu'elles ont pu être actives dans la production d'ignorance (Proctor, 1995), à laquelle les processus d'invisibilisation participent, en s'appuyant par exemple sur la réalisation d'études biaisées financées par ceux qui bénéficient du statu quo ou sur une volonté délibérée de verrouiller la création de nouvelles connaissances. La photographe inscrit et revendique ici, plus généralement, sa démarche dans une lutte pour le bien commun, anti-néolibérale dans laquelle la question environnementale semble occuper une place centrale. C'est la "grandeur " (Boltanski et Thévenot, 1987) revendiquée par l'artiste qui permet d'inscrire son action dans un régime d'engagement justifiable (Thévenot, 2011) : « dans [ce régime] qui prend appui sur les grandeurs, le bien tient à la capacité d'être qualifié pour sa participation à une certaine spécification du bien commun » (Thévenot, 2011). L'engagement de la photographe ici s'appuie sur l'idée théorique, comme le rappelle Chambefort-Kay (2017, p. 1), qu'« une image a un pouvoir de transformation de la société, qu'elle peut avoir une fonction, voire une responsabilité politique et sociale ». Le travail d'Anaïs Ondet, visant in fine à générer une prise de conscience, renvoie à la plus pure tradition documentaire. Sa démarche procède donc d'un choix puisant sa source dans un engagement chevillé au corps : être le médium d'un groupe invisible et générer chez le spectateur la curiosité voire l'empathie nécessaires à l'émergence de la connaissance.

\section{Le double usage du portrait : marqueur d'une trace et instrument de mise en mémoire}

«Un des aspects les plus essentiels, c'est que la photographie provoque et évoque autant la présence que l'absence » (Garrigues, 1998).

7 La photographie de manière générale, et le portrait en particulier, peuvent revêtir le statut de trace. Serres (2002) identifie « au moins quatre grandes significations de la trace, quatre points d'entrée pouvant donner lieu à autant de problématiques spécifiques [...] : 1) la trace comme empreinte, comme marque physique, [...] ; 2) la trace comme indice, comme "petite quantité", détail, avec le "paradigme indiciaire " proposé par Carlo Ginzburg ; 3) la trace comme mémoire, avec la question du document comme trace du passé, la connaissance par traces en histoire chez Ricœur, Paul Veyne ou Marc Bloch ; 4) la trace comme ligne, écriture et la problématique de la trace écrite, notamment chez Derrida, mais aussi la question actuelle du suivi des traces [...]» (p. 1). C'est à la fois la trace en tant qu'indice et la trace comme mémoire qui sont interrogées ici.

8 Plus que d'une simple légende, ces portraits sont accompagnés d'un véritable témoignage, recueilli dans le cadre d'un entretien semi-directif préalable à la réalisation du cliché. Portrait et témoignage participent à la production de connaissances, nouvelles et spécifiques. Il s'agit d'une part, de connaissances factuelles pouvant par exemple concerner l'organisation des territoires et leurs évolutions - et ce faisant - mettant en exergue l'évolution des possibilités d'exposition à des agents pathogènes ou encore éclairant la réalité de l'activité de travail. L'entretien réalisé avec Christian l'illustre particulièrement. L'individu, par sa présence, est un témoin et un 
acteur de l'histoire ( $C f$. Ricœur, 2000). Les évolutions qui concernent les pratiques agricoles et les réglementations (espacements entre les pieds de vigne par exemple, nombre de pieds à l'hectare) décidées par l'Union Européenne ( $c f$. réglementation du droit de plantation ${ }^{4}$ ) sont par exemples évoquées. Aussi, l'entretien semi-directif, parce qu'il fait parler, permet d'accéder au vécu et à la manière dont l'enquêté perçoit et se représente les choses. Ici, cette réalité est celle d'une activité de travail pathogène ${ }^{5}$, elle est celle du rapport au territoire ou encore du rapport à la maladie et des traces (visibles ou non) qu'elle laisse sur le corps et dans l'esprit. La trace est considérée par Ricœur comme "la racine commune au témoignage et à l'indice donnant toute son ampleur à la notion du document. [Enfin si], « la trace est de l'ordre du donné [...], le document est de l'ordre du construit » (Serres, 2012, p. 88).

9 En ce sens, le portrait photographique et le témoignage associé constituent « un fait résistant à l'effacement du passé »(Bretesché, 2014, p. 199). Ainsi, s'ils rendent visibles, s'ils contribuent à la connaissance et à la reconnaissance, ce «document » au sens de Ricœur, participerait aussi à une mise en mémoire des effets des agropesticides sur la santé humaine. Comme le précisent Bretesché et Ponnet (2012), en rappelant les travaux de Nora (1997), « la mémoire requiert des supports, des traces et des témoins ». Dans le cadre de cette démarche, le portrait photographique et le témoignage associé constituent tout à la fois le support mémoriel et la trace des impacts sanitaires des agropesticides sur la santé. Il précise également que «la mémoire est la vie, toujours portée par des groupes vivants et à ce titre, moins la mémoire est vécue de l'intérieur, plus elle a besoin de supports extérieurs et de repères tangibles d'une existence qui ne vit plus qu'à travers eux. D'où l'obsession de l'archive qui marque le contemporain, et qui affecte à la fois la conservation intégrale de tout le présent et la préservation intégrale de tout le passé ». La démarche photographique s'inscrit dans cette perspective. Créer les traces de ces impacts constitue aujourd'hui un enjeu important dans un contexte où l'oubli peut être organisé. À partir du cas des anciens sites uranifères, Bretesché (2014, p. 193) montre qu'en l'absence de ces traces, «un site tombe dans l'oubli notamment par un travail des différentes parties prenantes qui participent à la construction progressive de cet oubli. Ainsi, en l'absence de mémoire ravivée par les acteurs ou face à des conflits de mémoire, l'oubli peut être organisé de façon à effacer les traces d'un passé qui permettraient de rendre publics les signes tangibles d'un risque ». Le cas de la pollution générée par le Comptoir des Minéraux et des Matières (CMMP) à Aulnay-sous-Bois en Seine-Saint-Denis peut également être mentionné ici pour illustrer les enjeux de collecte des traces (Prost et al., 2020). Le réaménagement récent de cette usine de production et de transformation d'amiante, en activité entre 1938 et 1991 et qui a été à l'origine d'une pollution environnementale meurtrière, a soulevé d'importantes questions quant à la mise en mémoire de cette pollution et de ses impacts. C'est grâce à la mobilisation citoyenne et scientifique particulièrement active depuis le milieu des années 1990, que les traces de cette activité et ses conséquences sur la santé des travailleurs mais aussi des riverains, ont été collectées, et ce, malgré de nombreux obstacles rencontrés (refus d'accès aux archives de l'entreprise, nécessitant le recours à la Commission d'Accès aux Documents Administratifs entre autres). Bretesché (2014, p. 199) explique aussi que « localement, le recueil des souvenirs relatifs à l'exploitation de l'uranium et le sens donné au territoire soumis à la surveillance représentent des éléments tangibles susceptibles d'amorcer le travail de mémoire. Dans cette perspective, la trace permet de partir à la recherche d'un patrimoine non plus fondé sur les héros d'une autre époque mais sur les témoins 
ordinaires d'un territoire en mutation ». Avec sa démarche photographique, Anaiis Ondet participe à recueillir ces traces locales, dans une véritable démarche d'enquête.

\section{Le champ de maïs}

\section{Illustration 2}

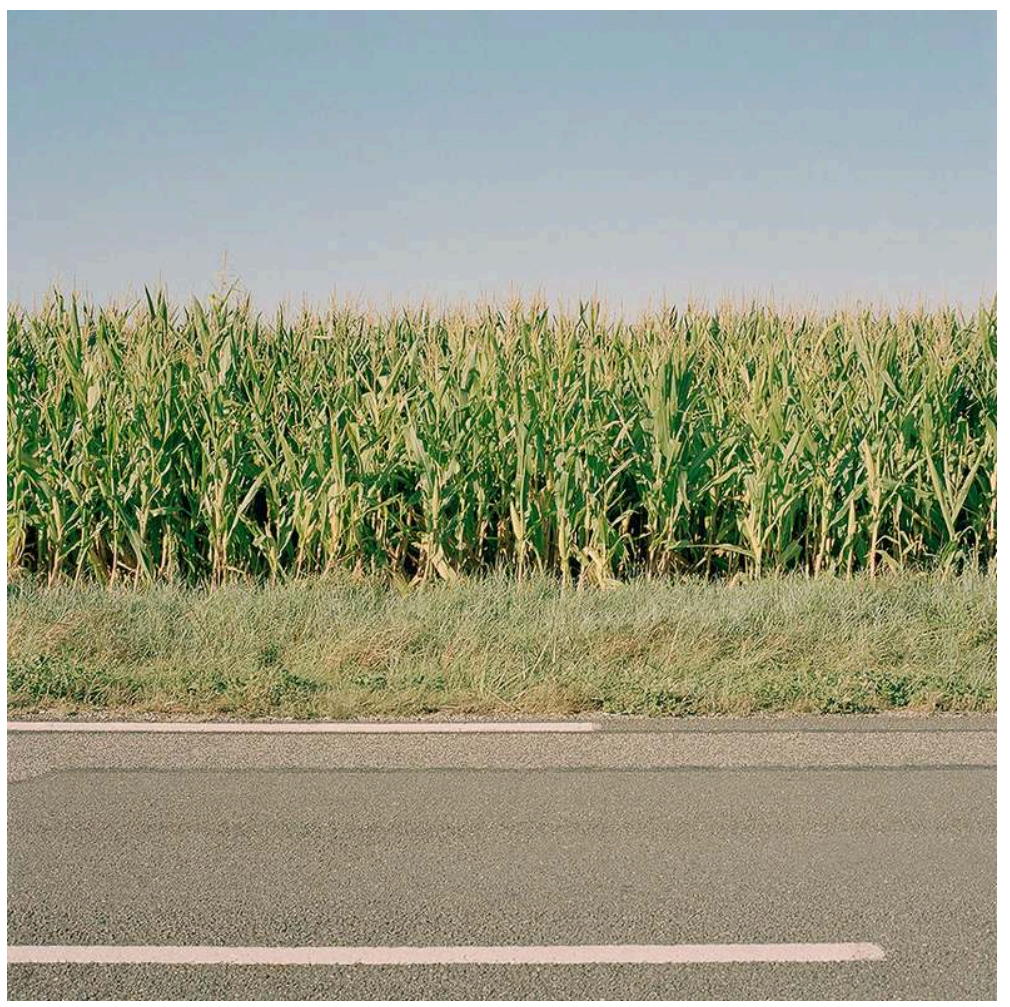

Auteure : Anaiis Ondet.

Proposée comme les autres au format carré, c'est une photographie dite de paysage. Elle est structurée par des lignes horizontales parallèles qui génèrent un effet de symétrie et de stabilité à l'image. Ces lignes divisent l'image en trois parties aux proportions identiques. Le ciel, très bleu sans nuage, un champ de maïs vert et une route bitumée grise et blanche. Les épis de maïs sont globalement de hauteur égale, équidistants. Toutes ces symétries et cet aspect épuré donnent au paysage une image aseptisée. La frontalité de cette image nous propose également un nouveau point de vue sur ce type de paysage évocateur d'une forme de banalité dans le contexte français. En effet, ce cliché nous pose en observateur passif et très stoïque. Nous sommes ainsi «forcés » de regarder, comme dans un face-à-face, une photographie représentant un paysage que l'on a plutôt l'habitude de voir rapidement, en mouvement et dont on ne questionne peu, voire pas, le contenu.

Qu'est-ce qu'un paysage? Qu'est ce qui fait le paysage? Questions soulevées par nombre de photographes et d'administrations politiques et culturelles des territoires. En témoignent les différentes commandes photographiques françaises. À ce titre, la Bibliothèque Nationale de France a produit en 2017 l'exposition Paysages français, Une aventure photographique, 1984-20176 proposant une rétrospective des commandes photographiques françaises de 1984 à 2017 sur la thématique de la photographie de paysage, des paysages français et de leur mutations. Ces questions se sont également posées lorsque j'ai commencé Les mauvaises herbes. Comment 
questionner la notion de paysage? Comment photographier les paysages de l'agriculture française ? Le paysage est produit en fonction des aménagements des territoires qui en sont fait mais également par rapport à l'histoire des représentations du paysage et à l'histoire de l'art selon le pays et l'époque. Ainsi actuellement en France le modèle agricole dominant est de l'ordre de la culture contrôlée, normée et dite "propre", c'est-à-dire sans adventices (mauvaises herbes) et les représentations qui en sont faites (iconographie que l'on retrouve dans les brochures touristiques et dans les outils de communication des collectivités territoriales notamment) sont également normées. On y retrouve des images très bucoliques, avec de belles lumières à l'aube ou au crépuscule, des paysages vallonnés et finalement très cultivés. Une seconde question s'est posée : comment photographier l'invisible? En effet, les produits phytosanitaires laissent peu, voire pas de traces physiques visibles sur la terre et les plantes. Avec un regard de néophyte, il m'était impossible de voir la différence entre un champ traité et un champs non traité, un champ bio et un champ en " conventionnel ». Au fur et à mesure de mes échanges avec des professionnels de l'agriculture j'ai appris à reconnaître certains marqueurs des traitements agrochimiques sur les champs et à comprendre aussi les motivations aidant certain à passer du champ conventionnel "propre » et régulier à une agriculture moins contrôlée dans laquelle la nature opère un peu plus librement. Il n'en reste pas moins qu'il me fallait traiter du paysage agricole conventionnel d'une certaine façon pour que mes images touchent un grand nombre de spectateurs et fassent écho à leur culture visuelle.

J'ai voulu ainsi jouer de ces représentations dites belles en créant des images reprenant les codes iconographiques dominants et en les opposants aux portraits durs des victimes de pesticides pour créer une ambivalence entre les deux images et pousser les spectateurs à la réflexion sur les représentations des paysages agricoles et ainsi sur l'agriculture française. Mes photographies possèdent, bien souvent, une trace visible de l'activité humaine afin de rappeler l'artificialité de cette nature cultivée. Mes séances de prises de vue sont des déambulations dans les campagnes où je m'arrête lorsque quelque chose dans le paysage me pointe ${ }^{7}$. Il s'agit paradoxalement d'une image belle et dérangeante qui vient me questionner. Je propose alors des paysages bucoliques et idéalisés de l'agriculture ainsi que des métaphores du passage de l'humain sur cette nature, créant ainsi des parallèles entre des images de terres labourées ou des plantes desséchées et les maladies des femmes et des hommes qui ont travaillé cette nature. Je questionne la trace de l'humain sur la nature dans le même temps que je questionne ma propre photographie et son caractère de trace. «La photographie nous arrête deux fois : la première fois en nous montrant cet objet ou cette scène que nous n'avions pas vus (...), en effet «l'habitude, remarque Harry Gruyært, tue le regard »-, la deuxième fois en nous obligeant à passer quelques secondes, voire quelques minutes à la regarder » (Soulages, 2017, p. 201).

Sontag (1977, p. 129) écrivait dans son œuvre Sur la photographie que «le regard photographique était la capacité à découvrir de la beauté dans ce que tout le monde voit mais néglige comme trop ordinaire ». Photographier l'ordinaire, c'est rendre visible ce qui est sous nos yeux mais que l'on ne voit plus. Photographier l'ordinaire, ce n'est pas ramener des images du lointain, de l'extraordinaire mais révéler notre quotidien pour permettre ainsi de l'observer sous un nouvel angle en nous posant en tant que spectateur, dans une nouvelle posture inhabituelle car passive et fixe. Réfléchir à des choses qui étaient tellement évidentes que l'on n'y prêtait pas attention. Photographier l'ordinaire est la motivation principale de mon travail : aller creuser les interstices du banal, y trouver un intérêt nouveau. En opposition à l'époque actuelle : décroissance, économie de la parole, économie du geste, économie de l'image animent ma pratique photographique. Sandor Krsana cité par Florence Delaye dans le film Sans Soleil de Chris Marker ${ }^{8}$ dit: "Après quelques tours du monde seule la banalité m'intéresse encore». La banalité, l'ordinaire comme base de travail, est quelque chose d'assez grand pour motiver 
une vie de photographies: chercher l'extraordinaire dans un environnement

familier, se concentrer sur l'ici et maintenant.

\section{Collecter les traces pathogènes}

10 Avec le projet Les mauvaises Herbes, l'artiste participe aussi à la construction de traces des caractéristiques d'un paysage qu'elle photographie à un moment donné de l'histoire. Le paysage fait l'objet de nombreux travaux en géographie. Nous retiendrons, dans le cadre de ce travail, deux sens : un sens « iconiste » et un sens « réaliste ». Dans le premier cas, "le paysage est une image, une représentation. C'est l'aspect d'une portion de territoire en tant qu'il est représenté par exemple par [...] une photographie » (Balibar, 2018, p. 11). Dans son acception « réaliste» le paysage « est un environnement réel, une chose du monde physique où nous nous trouvons » (Balibar, 2018, p. 11).

11 L'analyse du paysage de cette photographie est intéressante en ce sens qu'elle permet d'interroger les effets délétères des agropesticides - visibles ou non, reconnus, niés ou ignorés - sur l'environnement et ce faisant, sur la santé, à partir des traces laissées ou suggérées à travers la photographie. Son esthétique (couleur et alignement des épis de maïs, taille identique, etc.), « aseptisée » pour reprendre le vocable de la photographe, illustre ici une « agriculture contrôlée » notamment par l'usage d'agrotoxiques tels que les pesticides. Elle soulève ainsi des réflexions quant aux expositions professionnelles et environnementales à ces substances et à l'identification et la caractérisation des circonstances dans lesquelles ces expositions ont eu lieu. La dimension rétrospective de l'exercice participe de la mise en évidence complexe d'une relation entre exposition(s) et maladie. En effet, la plupart des maladies chroniques, qu'il s'agisse des cancers ou des maladies dégénératives comme celle de Parkinson ${ }^{9}$ dont il est question ici, résultent d'une multitude d'expositions à des substances toxiques, s'échelonnant dans un temps long. Analyser les circonstances d'exposition suppose ainsi la mise en regard d'une part des trajectoires des individus (professionnelles, résidentielles, etc.) et des trajectoires des territoires. C'est précisément en lien avec ces dernières que la démarche photographique présentée ici est intéressante. Ici la photographie «fige» les choses: un lieu caractérisé par la production de maïs. Cette photographie constitue désormais une trace de cette activité en ce lieu. La multiplication de photographies de ce type permet ainsi de disposer de connaissances sur les caractéristiques des lieux à un moment donné de l'histoire. C'est là où la complémentarité photographie / Sciences Humaines et Sociales apparait pertinente. Il devient alors possible de préciser des informations existantes dans différentes bases de données sur les territoires à l'aide d'éléments directement observés sur la photographie (types de cultures à un instant $\mathrm{T}$ par exemple). En complément, il pourrait donc être question de mobiliser des données sur les modes d'occupation des sols (Corine Land Cover $^{10}$ par exemple), pour reconstituer leur évolution spécifiquement pour le lieu où a été prise la photographie. La mission photographique de l'Institut National de l'Information Géographique et forestière (IGN) s'inscrit dans cette volonté de documentation des territoires et de leurs dynamiques. Ces prises de vue, dans leur cas, aériennes, «représente[ent] encore aujourd'hui la brique de base sur laquelle s'élabore la plupart des produits finaux : cartes papiers, modèles numériques de terrain, suivi des évolutions urbaines, rectification des parcelles cadastrales, etc.» (IGN, 2003, p. 22). Si la démarche de la photographe s'inscrit effectivement - en premier lieu - dans une démarche artistique, 
pour autant, elle peut également contribuer à documenter les caractéristiques des territoires.

12 Ainsi, la photographie permet de s'interroger sur les permanences et mutations que peuvent connaitre les territoires et les enjeux relatifs sous-jacents, en lien avec la mémoire ou la mise en mémoire. Cette photographie suggère des éléments relatifs aux sols (à leur qualité) notamment. À ce propos, Pomel, dans le cadre d'un ouvrage complet intitulé La mémoire des sols (2008), consacre un chapitre (le nº ${ }^{\circ}$ ) à la « mémoire des actions anthropiques». Mietton (2011) précise à ce titre, qu'en «reprenant notamment l'exemple des versants du Kilimandjaro étudiés très précisément, l'auteur montre comment les profils pédologiques enregistrent différentes actions anthropiques telles que les incendies, la déforestation, l'agriculture, le pastoralisme » (Mietton, 2011, p. 402). En effet, les pratiques relatives à l'utilisation de produits phytosanitaires en agriculture spécifiquement, dont il est question ici, laissent des traces, dans les paysages mais aussi dans les sols. En lien avec cette problématique, d'autres sources de données peuvent être mobilisées pour caractériser ces espaces de production. Ce sont par exemple, en agriculture, l'Indicateur de Fréquence des Traitements (IFT), qui est un «indicateur de suivi de l'utilisation des produits phytopharmaceutiques, à l'échelle d'une exploitation agricole ou d'un groupe d'exploitation » (Ministère de l'agriculture et de l'alimentation, 2019). Il faut toutefois garder à l'esprit que ces indicateurs peuvent parfois rendre compte d'un phénomène dont la réalité est assez éloignée. Ce faisant, les récits des individus collectés tout comme les fragments de paysage photographiés, dans le cadre de cette démarche, peuvent être mobilisés en complément, dans la production des connaissances sur un lieu, un territoire donné et les évolutions dont il est sujet. Mettre en regard ces différentes traces (résidus de pesticides dans les sols, photographies, souvenirs individuels) constituent aujourd'hui un enjeu important pour la connaissance des expositions professionnelles et environnementales réelles.

\section{S'arrêter sur l'ordinaire}

13 La photographie d'un paysage «banal »-car familier - est une démarche revendiquée par la photographe. Ce paysage ordinaire dans un référentiel agricole occidental est difficilement, à première vue, évocateur d'une nocivité potentielle. Cette banalité renforce in fine l'invisibilité de processus pathogènes conséquences - notamment - de l'emploi massif de pesticides dans l'agriculture intensive. Sans médiation documentaire de la part de l'artiste, il ne s'agit là que d'un simple champ de maïs - qui pourrait par ailleurs faire l'objet d'un traitement biologique- mais l'inscription de cette photographie dans un travail plus large et cohérent d'un point de vue thématique lui confère finalement son sens. La démarche est identique à celle du portrait : le cliché est le début d'une histoire qui par l'expression d'une idée oriente vers un message spécifique et stimule la connaissance. La lecture du cliché conduit ainsi à adopter une appréhension des processus par l'écologie des paysages. En effet, comme le rappellent Marty et al. (2006, p. 360), "si le paysage est conçu comme un système d'interactions entre les hommes et leur environnement, les processus ont au moins autant d'importance que la description des différentes configurations ». C'est bien la mise en exergue d'un processus multiscalaire aux temporalités multiples et emboitées qui permet de faire le lien entre un paysage banal et une maladie: des choix politiques, fortement dépendants d'un contexte historique, ont favorisé le développement d'une 
agriculture intensive et mécanique - que traduit le caractère rectiligne des cultures dans le paysage - basée sur l'utilisation massive d'agropesticides ayant pour unique but l'amélioration des rendements. C'est l'utilisation de ces produits chimiques qui génère, à un pas de temps pluriannuel voire pluridécennal, l'apparition de pathologies spécifiques de ces expositions. La maladie est la trace visible de la manière dont les hommes se sont saisis du paysage pour «exploit[er] ses ressources et modifi[er] intentionnellement ou non [ses] états, laiss[ant] des traces qui sont parfois évidentes parfois floues voire même inapparentes" (Marty et al., 2006, p. 359), comme les malades... Le caractère potentiellement pathogène de ce paysage est lui-même inapparent. Cette invisibilité physique (Thébaud-Mony, 2008) est renforcée par le caractère familier d'un paysage agricole : un champ de maïs en été dans le sud-ouest de la France. C'est un paysage ordinaire de la campagne française au sens conféré par Yves Luginbhül à la fin des années 1980. Godet (2010) nous permet d'inscrire ce paysage banal dans une nature surcomposée "calculée, provoquée et imposée par l'Homme». Définissant un degré croissant d'implication humaine, il considère ainsi que « [la nature peut être] recomposée, composée et surcomposée [et que] [...] par cette approche, la nature ordinaire est définie non pas comme un écotone fixe dans le temps et l'espace, mais bien comme une zone tampon mobile, au sein d'un espace dynamique car soumis à des forçages anthropiques et écologiques » (Godet, 2010, p. 300). Anaïs Ondet saisit une portion de la campagne occitane, à ses yeux, d'une grande banalité : son caractère ordinaire lui confère une nature si transparente que l'on oublie que les fleurs jaunes du colza remplaçaient peut-être le maïs l'an dernier au printemps.

Sans prendre le temps de s'arrêter sur ces lieux estompés par la force de l'habitude, il demeure de facto impossible d'entreprendre une lecture paysagère permettant autant la mise en lumière des processus présidant à leurs constructions que celle des potentielles externalités qu'ils engendrent. C'est tout l'intérêt de la démarche de la photographe ici : la révélation d'une nature familière. Elle souhaite dépasser le paysage comme apparence des choses, décor ou vitrine (Bertrand, 2000) et ambitionne ainsi d'engendrer cet "approfondissement sans fin des connaissances et, tout particulièrement de cette interactivité entre des éléments considérés comme différents, voire disparates et contradictoires : biophysiques et sociaux, économiques et culturels, patrimoniaux et prospectifs qui, combinés sur un même territoire, donnent naissance au paysage dans son apparente banalité quotidienne » (Bertrand, 2000, p. 62). La médiation de cette interactivité est une des propositions de l'artiste dans son cliché.

Reconnaître un paysage comme pathogène nécessite préalablement de le remarquer : c'est tout l'enjeu de la diffusion d'une image d'un paysage «banal». Ce paysage ordinaire est porteur d'un paradoxe entre un extrême contrôle de la nature par la technique et un «laisser aller » ou un « laisser faire»-pour reprendre les termes de Lelli et Paradis-Maindive (2000) - y compris empoisonner en silence la terre, ceux qui la travaillent voire ceux qui vivent à proximité. En levant partiellement le voile de la banalité et en ambitionnant de conférer à ce paysage façonné par l'agriculture une nature « extra-ordinaire ». À travers le message porté par la photographie, Anaïs Ondet engendre cette phase préliminaire essentielle pour passer du banal au remarquable dès lors qu'elle se pose « des questions issues de rencontres, d'idées, de confrontations, sur le devenir d'un territoire donné » (Lelli et Paradis-Maindive, 2000, p. 33). Sa volonté s'appuie une nouvelle fois sur la démarche documentaire qui inscrit son cliché dans un contexte et permet finalement d'exprimer certains enjeux invisibles de prime abord. 


\section{Remarques conclusives}

\section{BIBLIOGRAPHIE}

Asselin M., 2017. Monsanto : Une Enquête Photographique. Arles, Actes Sud, 156 p.

Balibar J., 2018. Du paysage représenté au paysage réel. Nouvelle Revue d'Esthétique [En ligne], vol. 2, n²2, p. 9-23. URL: https://www.cairn.info/revue-nouvelle-revue-d-esthetique-2018-2page-9.html - DOI: https://doi.org/10.3917/nre.022.0009

Bertrand G., 2000. Le paysage et la géographie : un nouveau rendez-vous. Treballs de la societat catalana de geografía, vol. 15, n50, p. 57-68.

Boltanski L., Thévenot L., 1987. Les économies de la grandeur. Cahiers du Centre d'études de l'emploi, Paris, PUF, 361 p.

Bretesché S., 2014. L'héritage patrimonial de l'uranium : de la trace à la mémoire disputée. L’homme \& la société, vol. 2, nº 192, p. 113-126.

Bretesché S., Ponnet M. 2012. Le risque au défi de la mémoire organisée : l'exemple de la gestion des mines d'uranium françaises. Vertigo. La revue électronique en sciences de l'environnement [En ligne], vol.12, n 1. URL: http://journals.openedition.org/vertigo/11992 - DOI: https:// doi.org/10.4000/vertigo.11992 
Chambefort-Kay K., 2017. Les Nouveaux modes d'engagement par la photographie en GrandeBretagne. Revue Française de Civilisation Britannique [En ligne], vol. 22, n 3. URL: http:// journals.openedition.org/rfcb/1487 - DOI: https://doi.org/10.4000/rfcb.1487

Freund G., 1974. Photographie et société. Paris, Editions du Seuil, 220 p.

Garrigues E., 1998. La photographie comme trace. Revista de dialectología y tradiciones populares, vol. 53, n 1, p.79-94.

Godet L., 2010. La « nature ordinaire » dans le monde occidental. L'Espace géographique, vol. 39, $n^{\circ} 4$, p. 295-308.

Henry H., 2017. Ignorance scientifique et inaction publique. Les politiques de santé au travail. Paris, Presses de Science Po, 264 p.

Honneth A., 2005. Invisibilité : sur l'épistémologie de la « reconnaissance ». Réseaux, vol. 1, $\mathrm{n}^{\circ}$ 129-130, p. 39-57.

Hunsmann M., Lysaniuk B., 2019. Faire entrer en résonance santé-travail et santéenvironnement. Une recherche-action sur les cancers d'origine professionnelle et environnementale dans la basse vallée du Rhône. Écologie \& politique, vol. 58, n 1, p. 83-106.

IGN., 2003. La photographie aérienne de l'IGN, depuis l'avion jusqu'à la BD Ortho. Géomatique expert [En ligne], $n^{\circ}$ 29. URL: http://www.geomag.fr/sites/default/files/28_37.pdf

INSERM (expertise collective), 2013. Pesticides : Effets sur la santé. Paris, Inserm, 1001 p.

Lelli L., Paradis-Maindive S., 2000. Quand le paysage ordinaire devient paysage remarqué. Sudouest européen, $n^{\circ} 7$, p. 27-34.

Marty P., Lepart J., Caplat P., 2006. Géographie et écologie des paysages : quelles relations? Bulletin de l'Association de Géographes Français, vol. 83, n 3, p. 355-367.

Mietton M., 2008. La mémoire des sols de Simon Pomel. Cahiers d'Outre-Mer, n² 243, p. 403-406.

Ministère de l'agriculture et de l'alimentation, 2019. Indicateur de Fréquence des Traitements phytosanitaires [En ligne]. URL: https://agriculture.gouv.fr/indicateur-de-frequence-detraitements-phytosanitaires-ift

Ministère de la transition écologique et solidaire \& Ministère de la cohésion des territoires et des relations avec les collectivités territoriales, 2018. CORINE Land Cover [Site en ligne]. URL: https:// www.statistiques.developpement-durable.gouv.fr/corine-land-cover-0

Nora P., 1997. Entre mémoire et histoire. La problématique des lieux. In Nora P., Les lieux de mémoire. Paris, Editions Gallimard, volume 1, p. 23-43.

Pomel S., 2008. La mémoire des sols. Bordeaux, Presses Universitaires de Bordeaux, 343 p.

Proctor R., N., 1995. The cancer wars: how politics shapes what we know and don't know about cancer. New York, Basic Books, 368 p.

Prost L., Lysaniuk B., Baron M., 2020. Réaménagement urbain, maladies industrielles et mémoires. L'usine d'amiante d'Aulnay-sous-Bois (Seine-Saint-Denis, France). Cahiers de Géographie du Québec, accepté, à paraître.

Ricœur P., 2000. La mémoire, l'histoire et l'oubli. Paris, Seuil, 675 p.

Serres A., 2002. Quelle(s) problématique(s) de la trace? Texte d'une communication prononcée lors du séminaire du CERCOR (actuellement CERSIC) [En ligne], 15 p. URL: https://

archivesic.ccsd.cnrs.fr/sic_00001397/document 
Serres A., 2012. La problématique de la trace à l'heure du numérique. Sens-Dessous, vol. $1, \mathrm{n}^{\circ}$ 10, p. 84-94.

Sontag S., 1977. Sur la photographie. Paris, Christian Bourgois Editeur, 280 p.

Soulages F., 2017 (2ème édition). Esthétique de la photographie, La perte et le reste. Paris, Armand Colin, $336 \mathrm{p}$.

Tardy J. N., 2007. Visibilité, invisibilité. Voir, faire voir, dissimuler. Hypothèses, vol. 1, nº 10, p. 15-24.

Thébaud-Mony A., 2008. Construire la visibilité des cancers professionnels. Une enquête permanente en Seine-Saint-Denis. Revue française des affaires sociales, $n^{\circ} 2$, p. 237-254.

Thévenot L., 2011. Grand résumé de L'Action au pluriel. Sociologie des régimes d'engagement [2006. Paris, Éditions La Découverte]. Sociologies [En ligne]. Grands résumés. URL: http:// journals.openedition.org/sociologies/3572

Voirol O., 2005. Les luttes pour la visibilité. Esquisse d'une problématique. Réseaux, vol. 1, $\mathrm{n}^{\circ} 129-130$, p. 89-121.

\section{NOTES}

1. Pour reprendre le titre d'un essai de Sigmund Freud.

2. De nombreux articles publiés par les chercheurs du Giscop93 (https://giscop93.univparis13.fr/) évoquent la succession d'obstacles à franchir pour faire reconnaitre sa maladie professionnelle comme telle.

3. Tableaux historiquement issus d'un compromis négocié entre des syndicats ouvriers et des organisations patronales comme le rappelle Emmanuel Henry (2017). La création d'un tableau est le reflet d'un point d'équilibre - à un moment donné - entre ces polarités.

4. Voir : https://eur-lex.europa.eu/legal-content/FR/TXT/PDF/?uri=CELEX: 32018R0274\&from=FRA

5. Le caractère pathogène de l'activité de travail renvoie à deux dimensions. Il est lié tout d'abord aux expositions délétères ayant cours durant l'exercice professionnel. Il renvoie ensuite aux expositions dites environnementales générées par le transfert de polluants en dehors de la sphère professionnelle et affectant potentiellement (par exemple) des riverains.

6. Voir : http://expositions.bnf.fr/paysages-francais/missions.php

7. La piqûre (ou « punctum ») chère à Roland Barthes.

8. Sans Soleil, Chris Marker, 1983.

9. L'Inserm précise l'étiologie et les facteurs de risques de la maladie : « en dehors des rares formes familiales liées à des mutations de gènes majeurs, [...] cette pathologie est généralement considérée comme une maladie multifactorielle résultant dans la majorité des cas de l'effet de facteurs multiples, qu'ils soient génétiques ou environnementaux » (Inserm, 2013, p.435). D’après « la méta-analyse de Van der Mark et coll., 2012, le risque de maladie de Parkinson était 1,62 (IC95\% [1,40-1,88]) fois plus élevé chez les personnes exposées aux pesticides au cours de leur vie » (Inserm, 2013, p.447).

10. Le base de données Corine Land Cover « est produite par 39 États européens dans le cadre programme européen de surveillance des terres de Copernicus, piloté par l'Agence européenne pour l'environnement [...]. Cet inventaire biophysique de l'occupation des terres fournit une photographie complète de l'occupation des sols, à des fréquences régulières. [...] Elle est issue de l'interprétation visuelle d'images satellitaires » (Ministère de la transition écologique et solidaire / Ministère de la cohésion des territoires et des relations avec les collectivités territoriales, 2018). 


\section{RÉSUMÉS}

La série documentaire Les mauvaises herbes d'Anaïs Ondet met en scène des portraits de femmes et d'hommes atteint.e.s de maladies liées à l'utilisation d'agropesticides et des paysages d'une campagne ordinaire où l'aspect pathogène demeure - de prime abord - invisible. L'exploitation de deux clichés de cette série offre l'occasion, dans le cadre d'un dialogue sciences-sociales/ photographie, de questionner les notions d'(in)visibilité, de reconnaissance, de mémoire, de trace et de banalité. L'expression de la photographe renvoie à un engagement profond visant à placer dans la lumière des victimes que l'on entend pas et à s'arrêter sur des paysages que l'on ne remarque plus.

Anaïs Ondet's documentary series Les mauvaises herbes features portraits of women and men suffering from diseases related to the use of agro-pesticides and landscapes cliché of an ordinary countryside where the pathogenic aspect remains - at first sight - invisible. The use of two photographs from this series offers the opportunity, in the context of a dialogue between social sciences and photography, to question the notions of (in)visibility, recognition, memory, trace and banality. The photographer's expression refers to a deep commitment to highlight victims that we do not hear and to focus on landscapes that we no longer notice.

\section{INDEX}

Mots-clés : portrait, paysage, maladie, visibilité, reconnaissance

Keywords : portrait, landscape, disease, visibility, recognition

\section{AUTEURS}

\section{BENJAMIN LYSANIUK}

Benjamin Lysaniuk, Benjamin.Lysaniuk@cnrs.fr, est chargé de Recherche à l'IRD (mise à disposition par le CNRS) et membre de l'UMR Prodig. Il a récemment publié - Lysaniuk B., Cely-García M. F., Mazzeo A., et al., 2020. Where are the landfilled zones? Use of historical geographic information and local spatial knowledge to determine the location of underground asbestos contamination in Sibaté (Colombia). Environmental Research, vol. 191, 110182. DOI: https://doi.org/10.1016/j.envres.2020.110182

- Cely-García M. F., Lysaniuk B., Pasetto R., 2020. Ramos-Bonilla J.R., 2020. The challenges of applying an Activity-Based Sampling methodology to estimate the cancer risk associated with asbestos contaminated landfilled zones. Environmental Research, vol. 181, 108893. DOI: https:// doi.org/10.1016/j.envres.2019.108893

- Prost L., Lysaniuk B., Baron M., 2020. Réaménagement urbain, maladies industrielles et mémoires. L'usine d'amiante d'Aulnay-sous-Bois (Seine-Saint-Denis, France). Cahiers de Géographie du Québec, accepté, à paraître.

\section{ANAÏS ONDET}

Anaïs Ondet est photographe indépendante et auteure. 


\section{LÉA PROST}

Léa Prost est doctorante et ATER à l'UFR Lettres Langues et Sciences Humaines de l'Université Paris Est Créteil. Elle a récemment publié :

- Prost L., Lysaniuk B., Baron M., 2018. Mémoires de maladies industrielles à Aulnay-sous-Bois. Valorisation / dévalorisation du territoire, entre reconnaissance et effacement. Actes du colloque du Collège International des Sciences du Territoire (CIST) du 22 au 24 mars 2018, Rouen.

- Prost L., Lysaniuk B., Baron M., 2020. Réaménagement urbain, maladies industrielles et mémoires. L'usine d'amiante d'Aulnay-sous-Bois (Seine-Saint-Denis, France). Cahiers de Géographie du Québec, accepté, à paraître.

- Prost L.,2021.Reconstituer les trajectoires résidentielles de patients atteints de Lymphomes Non-Hodgkiniens pour appréhender les réalités des risques d'exposition cancérogène (basse vallée du Rhône). Actes du colloque du Collège International des Sciences du Territoire (CIST) du 18 au 21 novembre 2021, accepté, à paraître. 